\title{
Linked annotations: a middle ground for manual curation of biomedical databases and text corpora
}

Tatyana Goldberg ${ }^{1,2}$, Shrikant Vinchurkar ${ }^{1}$, Juan Miguel Cejuela ${ }^{1}$, Lars Juhl Jensen ${ }^{3 *}$, and Burkhard Rost ${ }^{1 *}$

1 Bioinformatics \& Computational Biology, Department of Informatics, Technical University of Munich (TUM), 85748 Garching, Germany

${ }^{2}$ TUM Graduate School, Center of Doctoral Studies in Informatics and its Applications (CeDoSIA), 85748 Garching, Germany

${ }^{3}$ Novo Nordisk Foundation Center for Protein Research, Faculty of Health and Medical Sciences, University of Copenhagen, 2200 Copenhagen N, Denmark

* To whom correspondence should be addressed: lars.juhl.jensen@cpr.ku.dk or assistant@rostlab.org

\begin{abstract}
Annotators of text corpora and biomedical databases carry out the same labor-intensive task to manually extract structured data from unstructured text. Tasks are needlessly repeated because text corpora are widely scattered. We envision that a linked annotation resource unifying many corpora could be a game changer. Such an open forum will help focus on novel annotations and on optimally benefiting from the energy of many experts. As proof-of-concept, we annotated protein subcellular localization in 100 abstracts cited by UniProtKB. The detailed comparison between our new corpus and the original UniProtKB annotations revealed sustained novel annotations for $42 \%$ of the entries (proteins). In a unified linked annotation resource these could immediately extend the utility of text corpora beyond the text-mining community. Our example motivates the central idea that linked annotations from text corpora can complement database annotations.
\end{abstract}

\section{Background}

The natural language processing (NLP) and biomedical research communities have in common that they invest great effort into making high-quality manual annotation of biomedical literature. The focus and the annotation strategies of the two communities have, however, differed so much that collaborations remained stunningly limited. Most text corpora contain detailed markup of only a few types of entities and relationships in a limited number of abstracts or articles [Neves, 2014] (with exceptions such as the CRAFT corpus [Verspoor et al., 2012]). In contrast, manually curated databases such as Swiss-Prot/UniProtKB [UniProt Consortium, 2014] aim at annotating each entity with a wide range of information extracted from literature, but with less focus on the text structure. 
We envision linked annotations as a possible middle ground for the two important strategies to curate literature that could synergistically link the efforts of two distinct communities. By connecting the annotations of different types of entities and relationships annotated in existing and future corpora, a linked annotation resource could be constructed, which would have much greater coverage and diversity of annotations than any existing text corpus. Such a corpus would be valuable to NLP researchers and database curators alike.

Here, we present a case study on protein subcellular localization to demonstrate that the corpus annotation strategy can improve database annotation. The localization of a protein is one aspect of protein function and therefore constitutes one of the three hierarchies to capture protein function employed by the Gene Ontology (GO) [Ashburner et al., 2000].

\title{
The LocText corpus
}

We assembled a corpus of 100 PubMed abstracts referenced by UniProtKB. We focused on three model organisms: Homo sapiens (50 entries), Saccharomyces cerevisiae (baker's yeast with 25 entries), and Arabidopsis thaliana as a plant (25 entries). We used 46 of the 100 abstracts to develop our annotation guidelines that are available at https://www.tagtog.net/-corpora/loctext.

\section{A cysteine-rich receptor-like kinase NCRK and a pathogen-induced protein kinase RBK1 are Rop GTPase interactors.}

\begin{abstract}
In plants, Rop/Rac GTPases have emerged as central regulators of diverse signalling pathways in plant growth and pathogen defence. When active, they interact with a wide range of downstream effectors. Using yeast two-hybrid screening we have found three previously uncharacterized receptor-like protein kinases to be Rop GTPase-interacting molecules: a cysteine-rich receptor kinase, named NCRK, and two receptor-like cytosolic kinases from the Arabidopsis RLCK-VIb family, named RBK1 and RBK2. Uniquely for Rho-family small GTPases, plant Rop GTPases were found to interact directly with the protein kinase domains. Rop4 bound NCRK preferentially in the GTP-bound conformation as determined by flow cytometric fluorescence resonance energy transfer measurements in insect cells. The kinase RBK1 did not phosphorylate Rop4 in vitro, suggesting that the protein kinases are targets for Rop signalling. Bimolecular fluorescence complementation assays demonstrated that Rop4 interacted in vivo with NCRK and RBK1 at the plant plasma membrane. In Arabidopsiș protoplasts, NCRK was hyperphosphorylated and partially co-localized with the small GTPase RabF2a in endosomes Gene expression analysis indicated that the single-copy NCRK gene was relatively upregulated in vasculature, especially in developing tracheary elements. The seven Arabidopsis RLCK-VIb genes are ubiquitously expressed in plant development, and highly so in pollen, as in case of RBK2. We show that the developmental context of RBK1 gene expression is predominantly associated with vasculature and is also locally upregulated in leaves exposed to Phytophthora infestans and Botrytis cinerea pathogens. Our data indicate the existence of cross-talk between Rop GTPases and specific receptor-like kinases through direct molecular interaction.
\end{abstract}

Figure 1. Curation of protein subcellular localization. The simplified tagtog web interface shown assisted in the manual annotation of the corpus (abstract of [Molendijk et al., 2008]). Colours highlight names of organisms (yellow), genes/proteins (green), and localization terms (magenta). Linking the Arabidopsis protein RabF2a (UniProtKB ID: RAF2A_ARATH) to endosomes adds a novel annotation to UniProtKB. 
Two of us (TG \& SV) then annotated the remaining 54 abstracts. The two annotations agreed at $F_{1}=94 \%$ for entities and at $F_{1}=80 \%$ for relationships. We normalized protein names to UniProtKB and localizations to $\mathrm{GO}$ identifiers. The resulting corpus contains 306 annotated relationships in 201 different UniProtKB proteins with $48 \mathrm{GO}$ distinct localization terms. All annotations were made within the framework of the tagtog system (Figure 1) [Cejuela et al., 2014; http://tagtog.net] and Reflect was used to aid protein name normalization [Pafilis et al, 2009; http://reflect.ws]. The corpus is available for download at https://www.tagtog.net/-corpora/loctext under the Creative Commons Attribution 4.0 (CC-BY 4.0) license.

\section{Corpus provides novel annotations}

Linked annotations from text corpora can complement database annotations only if manual corpus annotations identify relationships not captured by existing databases. Therefore, all our annotations were done from scratch without using database annotations. Comparing our "from scratch" annotations with those from UniProtKB revealed important novelty added by our text corpus.

Table 1. Localization annotations in our corpus and in UniProtKB.

\begin{tabular}{lllllll}
\hline Category & \multicolumn{2}{l}{ Existing } & \multicolumn{2}{c}{ More detailed } & Novel \\
Citing protein & Yes & No & Yes & No & Yes & No \\
\hline Human & 29 & 15 & 1 & 1 & 14 & 13 \\
Budding yeast & 22 & 14 & 5 & 3 & 6 & 15 \\
Arabidopsis & 19 & 7 & 5 & 2 & 6 & 7 \\
Other & 2 & 9 & 0 & 0 & 0 & 6 \\
\hline Subtotal & 72 & 45 & 11 & 6 & 26 & 41 \\
\hline Total & 117 & & 17 & & 67 & \\
\hline
\end{tabular}

The table categorizes the corpus relationships by organism relative to whether they represent existing annotations in UniProtKB, more detailed annotations, or truly novel annotations. It further subdivides the counts based on whether or not the relationships involve UniProtKB proteins that cite the abstract. 
We found novel or more detailed localization annotations with respect to UniProtKB for 84 of 201 (42\%) proteins in 34 abstracts (Table 1); for example, Arabidopsis RabF2a (UniProtKB entry RAF2A_ARATH) is localized to endosomes (Figure 1). We found that for over half of these proteins with additional annotations (47/84=56\%) UniProtKB did not cite the abstracts. This is likely explained by the way proteins are annotated, one protein at a time: if a curator works on one protein and an abstract mentions also the localization of another, which is not the focus of curator, the localization of the latter might not be annotated.

\section{Perspectives}

Our case study clearly showed that corpora containing manual annotations of the subcellular localization of proteins are able to contribute novel information to curated databases such as UniProtKB. Notably, this is even true in the worst-case example when limiting annotations only to abstracts of articles that have already been utilized by the database curators. We expect our findings to generalize to most types of protein annotation, including disease associations and tissue expression.

Today databases avoid the trouble of integrating these annotations, because most text corpora are too limited in size and scope. Having the corpus developers combine their annotations into a single, unified linked annotation resource could thus be an important step towards integration of corpus annotations into databases, thus making them to richer data collection systems. Even before integration with databases happens, it will be possible for researchers to use semantic web technologies to combine the information in the linked annotation resource with that in existing databases, since UniProtKB and many other databases are already Resource Description Framework (RDF) compliant.

We envision a linked annotation resource to continuously grow, supported by annotation tools making it easy for corpus developers to link future annotations; for example, through a standard JSON format. Not all linked annotations need to be made manually, though. Including also results from automatic text mining pipelines would help address the challenge of the prohibitively high costs of large-scale manual annotation [Baumgartner et al., 2007]. Associations extracted from both open and non-open access journals can be linked, as redistribution of extracted facts is not prohibited by most publishers' licenses. 


\section{Acknowledgments}

Funding: Alexander von Humboldt Foundation through German Federal Ministry for Education and Research, Ernst Ludwig Ehrlich Studienwerk, and the Novo Nordisk Foundation Center for Protein Research (NNF14CC0001).

\section{References}

Ashburner M, Ball CA, Blake JA, Botstein D, Butler H, Cherry JM, Davis AP, Dolinski K, Dwight SS, Eppig JT, Harris MA, Hill DP, Issel-Tarver L, Kasarskis A, Lewis S, Matese JC, Richardson JE, Ringwald M, Rubin GM, Sherlock G (2000). Gene ontology: tool for the unification of biology. Nat Genet. 25:25-29.

Baumgartner WA, Cohen KB, Fox LM, Acquaah-Mensah G, Hunter L (2007). Manual curation is not sufficient for annotation of genomic databases. Bioinformatics 23(13):i41-i48.

Cejuela JM, McQuilton P, Ponting L, Marygold SJ, Stefancsik R, Millburn GH, Rost B; FlyBase Consortium (2014). tagtog: interactive and text-mining-assisted annotation of gene mentions in PLOS full-text articles. Database 2014:bau033.

Molendijk AJ, Ruperti B, Singh MK, Dovzhenko A, Ditengou FA, Milia M, Westphal L, Rosahl S, Soellick TR, Uhrig J, Weingarten L, Huber M, Palme K (2008). A cysteine-rich receptor-like kinase NCRK and a pathogen-induced protein kinase RBK1 are Rop GTPase interactors. Plant J. 53:909-923.

Neves M (2014). An analysis on the entity annotations in biological corpora. F1000Res. 3:96.

Pafilis E, O'Donoghue SI, Jensen LJ, Horn H, Kuhn M, Brown NP, Schneider R (2009). Reflect: augmented browsing for the life scientist. Nat Biotechnol. 27:508-510.

UniProt Consortium (2014). Activities at the Universal Protein Resource (UniProt). Nucleic Acids Res. 42:D191-D198.

Verspoor K, Cohen KB, Lanfranchi A, Warner C, Johnson HL, Roeder C, Choi JD, Funk C, Malenkiy Y, Eckert M, Xue N, Baumgartner WA Jr, Bada M, Palmer M, Hunter LE (2012). A corpus of full-text journal articles is a robust evaluation tool for revealing differences in performance of biomedical natural language processing tools. BMC Bioinformatics 13:207. 